\title{
Preliminary Application of Synthetic Computed Tomography Image Generation from Magnetic Resonance Image Using Deep-Learning in Breast Cancer Patients
}

\author{
Wan Jeon ${ }^{1, *}$, Hyun Joon An ${ }^{2, *}$, Jung-in Kim ${ }^{2,3}$, Jong Min Park ${ }^{2,3}$, Hyoungnyoun Kim ${ }^{4}$ Kyung Hwan Shin ${ }^{2,3,5}$, \\ Eui Kyu Chie ${ }^{2,3,5}$ \\ ${ }^{1}$ Department of Radiation Oncology, Dongnam Institute of Radiological and Medical Sciences, Busan, Korea; ${ }^{2}$ Department of Radiation Oncology, Seoul \\ National University Hospital, Seoul, Korea; ${ }^{3}$ Institute of Radiation Medicine, Medical Research Center, Seoul National University, Seoul, Korea; ${ }^{4}$ GenAI Inc., \\ Seoul, Korea; ${ }^{5}$ Department of Radiation Oncology, Seoul National University College of Medicine, Seoul, Korea.
}

\section{Original Research}

Received May 27, 2019

Revision November 25, 2019

Accepted December 13, 2019

Corresponding author: Eui Kyu Chie

Department of Radiation Oncology, Seoul National University Hospital, 101 Daehak-ro, Jongno-gu, Seoul 03080, Korea

Tel: $+82-2-2072-3705$

Fax: +82-2-765-3317

E-mail: ekchie93@snu.ac.kr

*Wan Jeon and Hyun Joon An contributed equally to this work.

This is an Open-Access article distributed under the terms of the Creative Commons Attribution NonCommercial License (http://creativecommons.org/ licenses/by-nc/3.0) which permits unrestricted noncommercial use, distribution, and reproduction in any medium, provided the original work is properly cited.

Copyright $\odot$ 2019The Korean Association for Radiation Protection

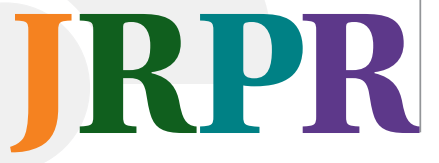

Background: Magnetic resonance (MR) image guided radiation therapy system, enables real time MR guided radiotherapy (RT) without additional radiation exposure to patients during treatment. However, MR image lacks electron density information required for dose calculation. Image fusion algorithm with deformable registration between MR and computed tomography (CT) was developed to solve this issue. However, delivered dose may be different due to volumetric changes during image registration process. In this respect, synthetic CT generated from the MR image would provide more accurate information required for the real time RT.

Materials and Methods: We analyzed 1,209 MR images from 16 patients who underwent MR guided RT. Structures were divided into five tissue types, air, lung, fat, soft tissue and bone, according to the Hounsfield unit of deformed CT. Using the deep learning model (U-NET model), synthetic CT images were generated from the MR images acquired during RT. This synthetic CT images were compared to deformed CT generated using the deformable registration. Pixelto-pixel match was conducted to compare the synthetic and deformed CT images.

Results and Discussion: In two test image sets, average pixel match rate per section was more than $70 \%$ (67.9 to $80.3 \%$ and 60.1 to $79 \%$; synthetic CT pixel/deformed planning CT pixel) and the average pixel match rate in the entire patient image set was $69.8 \%$.

Conclusion: The synthetic CT generated from the MR images were comparable to deformed $\mathrm{CT}$, suggesting possible use for real time RT. Deep learning model may further improve match rate of synthetic $\mathrm{CT}$ with larger MR imaging data.

Keywords: Deep Learning, Image Guided Radiotherapy, MRI, Synthetic CT

\section{Introduction}

Image-guided radiotherapy aims to increase the accuracy of radiation therapy to deliver the optimal dose to the patient. MRIdian, a magnetic resonance (MR) image guided radiation therapy system (Viewray Inc., Oakwood, OH, USA), not only prevents additional radiation exposure other than the dose delivered to the patient, but also enables adaptive treatment in real-time by taking into account of the changes in both tu- 
mor and surrounding organ at risk $[1,2]$. However, MR imaging is fundamentally different from computed tomography (CT) imaging. Radiation dose calculation is not possible as the MR image is devoid of electron density information required for dose calculation [3]. To solve this problem, image matching algorithm with deformable registration between MR and CT was developed. However, volumetric changes during image registration limits calculation of actual radiation dose delivered $[4,5]$. In this respect, synthetic CT generated from the MR image would provide information required for the real time RT planning with actual dose calculation. For this purpose, we aimed to develop a synthetic CT generation algorithm using the deep learning method. MR images obtained from the MR imaging radiotherapy system was compared to deformed planning CT to build model for synthetic CT image generation [6-8]. Generated synthetic CT images were then compared to deformed CT.

\section{Materials and Methods}

We analyzed 1,209 MR images from 16 patients who underwent MR image guided radiotherapy from December 2015 to August 2017. This study was approved by the Institu- tional Review Board prior to initiation (approval No. 1708051-876).

Each MR image was learned through U-net Convolutional Networks for Biomedical Image Segmentation model [9]. In the MR images, blurred image slices at edge along the axial dimension were excluded from each patient dataset. CT images were aligned to the corresponding MR images through deformable registration provided by ViewRay treatment planning system. No manual correction was performed. After registration process, these deformed CT (dCT) images were resampled to the same size of MR images. The accuracy of image registration was visually compared by the boardcertified radiation oncologist, and it was excluded from the dataset when the mismatch occurred in the outer and inner contour of patient body. Algorithm generation and validation were performed for patients with deformed image matching compared to pretreatment CT for each patient. The relationship between MR images and CT images was evaluated according to the CT Hounsfield unit (HU) reference values, such as air (-100 to -900$)$, bone (150 to 1,200$)$, fat (-400 to 10$)$, lung ( -900 to 400$)$, and soft tissues (-10 to 150). Based on the above criteria, deformed CT (dCT) was generated from the MR image. Since the magnitude of the MR image may vary depend-
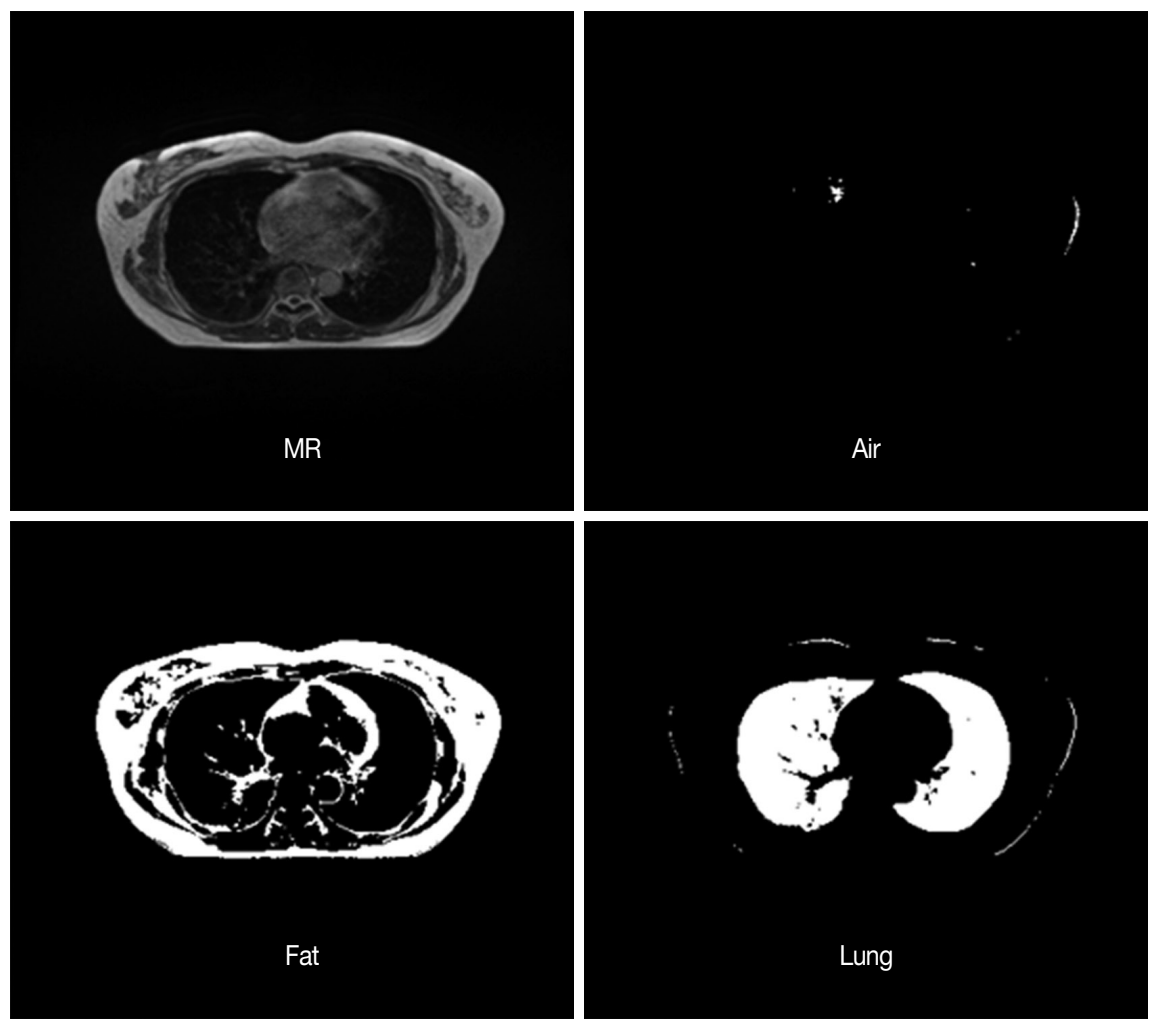

Fig. 1. Based on the CT HU, dCT images separated into five tissues.
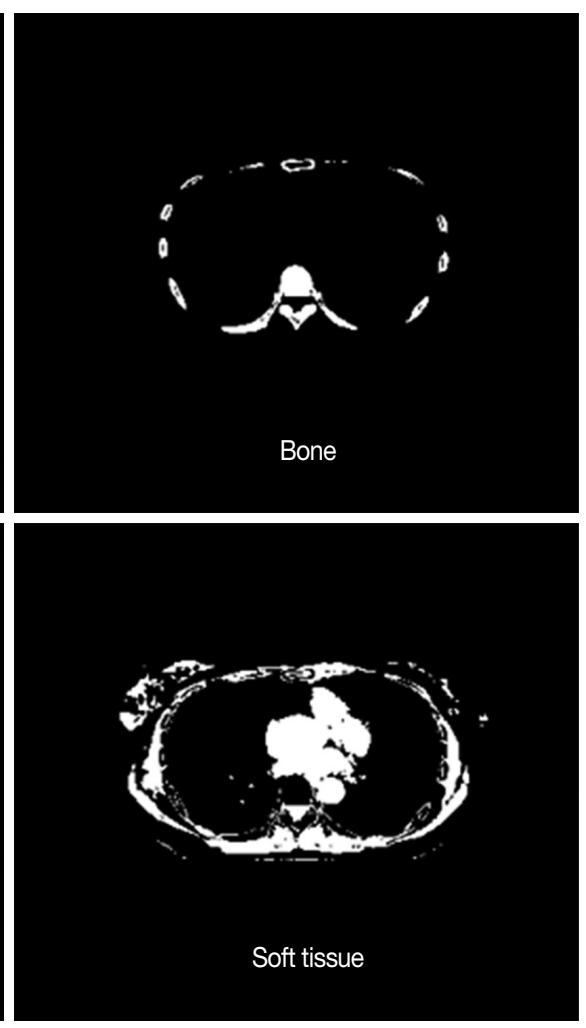
ing on the imaging device, the local maximum value for each image and the global maximum value for the pixel acquired from the MR image of the entire specific patient data was obtained, and the normalization process was performed therefrom. Computed tomography images were pre-processed to remove the outer part of the CT image, such as frames and immobilization devices based on the area of the MR image. DCT images were then separated into five tissue types based on the CT number (Figure 1). As the CT number is a continuous value, noise was included. A batch program was used to segregate dCT images. Separated images were then synthesized and reconstructed to build dCT images separated into five different tissues, dCT (5 level), and then compared with original MR images, and original dCT images (Figure 2).

\section{Patient's Characteristics}

Sixteen patients diagnosed with breast cancer and received MR guided breast radiotherapy were analyzed for synthetic CT generation. MR images of each patient covered the entire lung, and axial images were acquired at $3 \mathrm{~mm}$ intervals. A total of 1,209 MR images were obtained from 16 patients.

Deep learning was performed based on the U-net model using MR images of all patients. Fourteen patients were assigned training sets and 2 patients were validated with image data.

\section{Deep learning based on U-net model}

We generated five learning models that map five dCT tis-
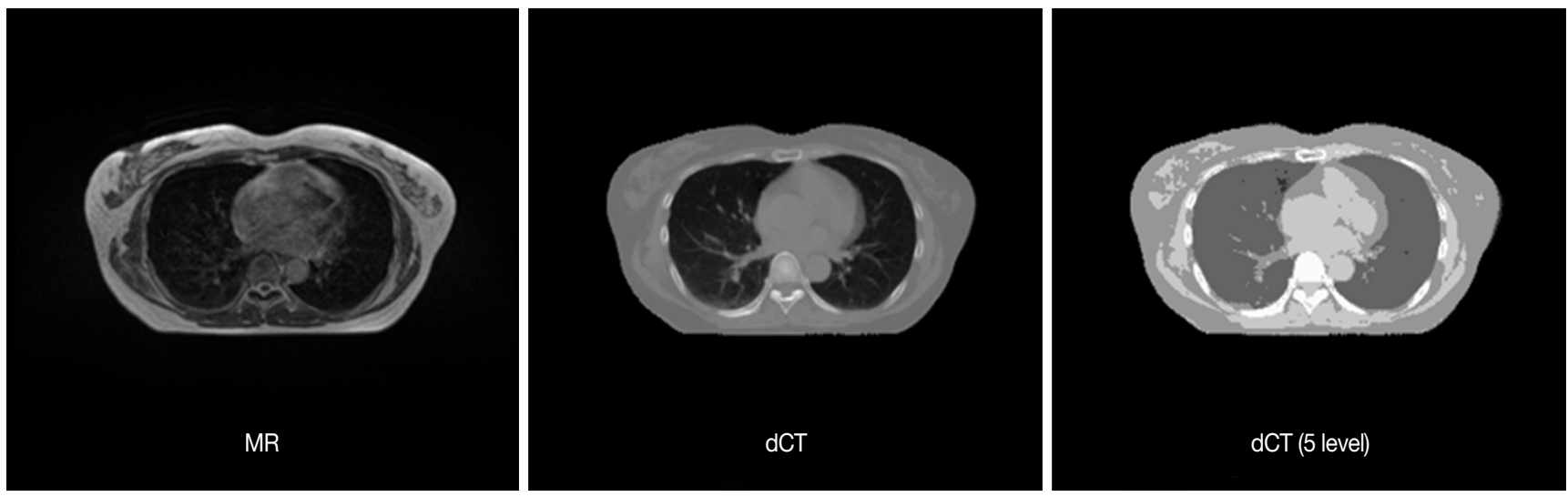

Fig. 2. MR images, dCT images, and dCT images of 5 different tissues.

Input image
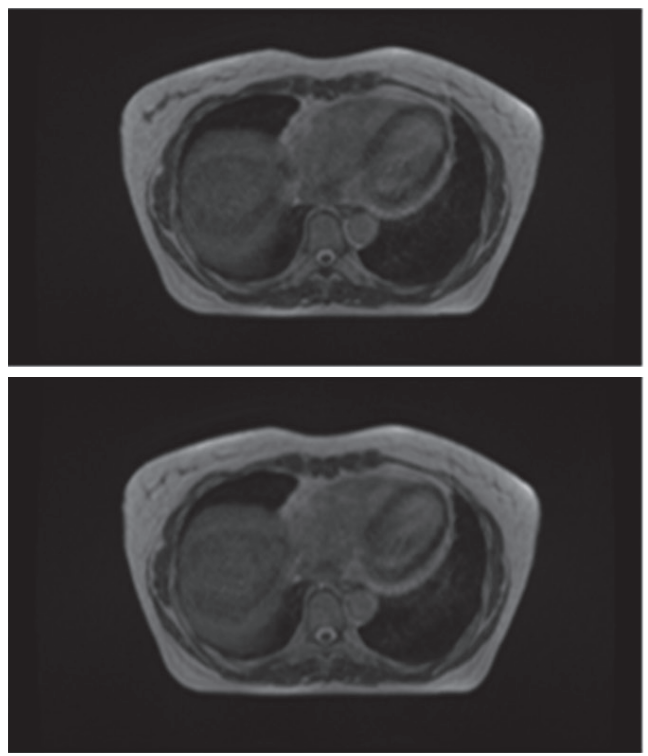
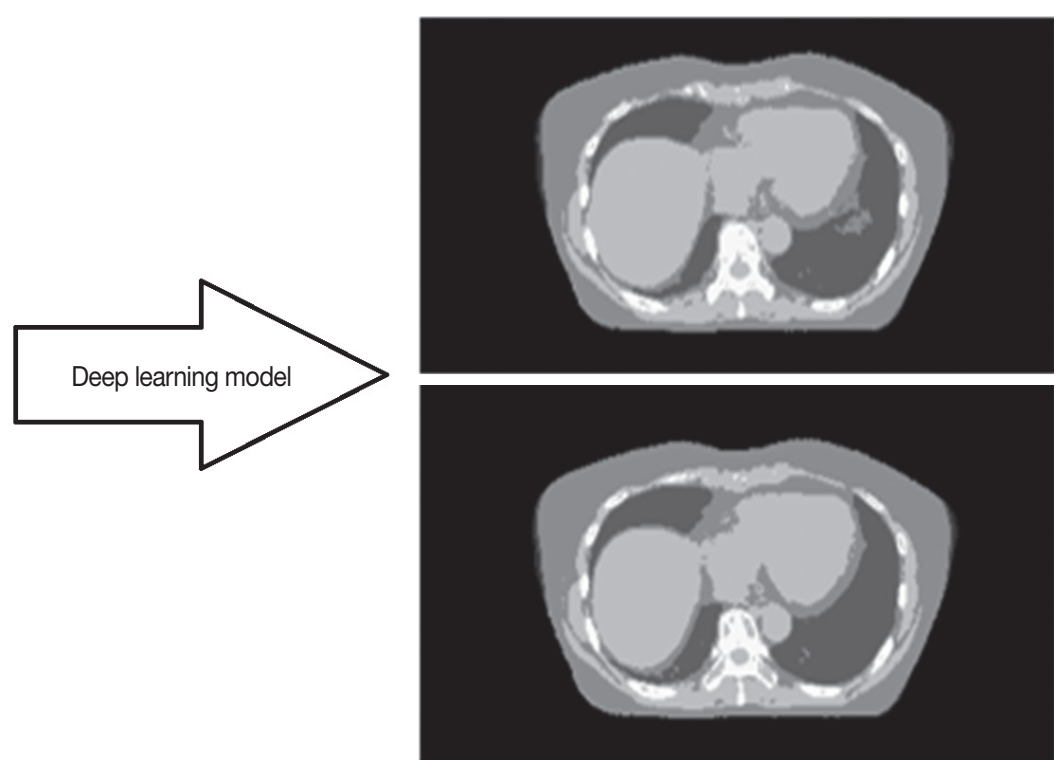

Fig. 3. Deep learning model (U-net) that converts MR image to dCT image. 
sue images based on one MR image. It is applied to the U-net model corresponding to the segmentation technique among the deep learning models and maps the MR image and CT regions of the tissue (Figure 3) Deep learning is based on the Keras framework with Theano Backend. In the database of 16 patients, 1,209 MR-dCT pairs were created, and the images were learned. The MR image is used as the input image, and the dCT image is designated as the mask image to model the relationship between the input and mask images. For lack of study data, the pre-trained network trained by MR/ CT data set (https://github.com/ZFTurbo/ZF_UNET_224_ Pretrained_Model/) was used according to transfer learning algorithm. The pre-trained networks were trained using the Adam optimizer with a learning rate of 0.0001 and dropout rate of 0.05 . The binary cross-entropy was used as loss function. The batch size is set to 20 .

The size of the image applied to the training was adjusted to $224 \times 224$ pixels, and the batch size was updated to 16 when the lowest loss value is within 200 epochs. To evaluate the performance of the learned model, we added air, lung, fat, soft tissue and bone data from more patients. In the training, we used the Dice coefficient as the pixel match rate to measure the loss function and the error for the backpropaga- tion algorithm as shown below.

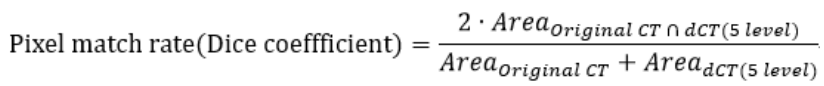

Using all five learned models, we generated five output mask images from arbitrary input MR images. When the mask images are combined and adjusted by each gray-scale, a synthetic CT as shown in Figure 4 is derived. Image match was evaluated by comparing the pixel values in the composite CT generated by the deep learning method to the original CT. Match rate of the image was calculated from pixel-to-pixel match of the synthetic CT against dCT (Figure 5).

\section{Results and Discussion}

The first validation was carried out for two out of 16 patients, and the pixel match rate for each patient was as shown in Table 1 . For the test patient 1 , a maximum of $80.3 \%$, at least $67.9 \%$, and an average of $76.4 \%$ match rate were found in 72 images. Test Patient 2 had a maximum match rate of $79 \%$, minimum $60.1 \%$ and average $73.5 \%$ in 64 images.

We then analyzed the pixel match rate of the synthetic CT for all 16 patients. Average pixel match rate was $68.7 \%$, stan-
Original MR

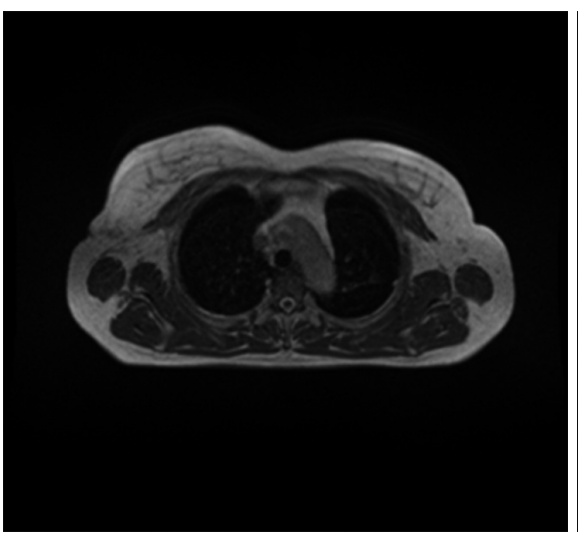

Mask_soft

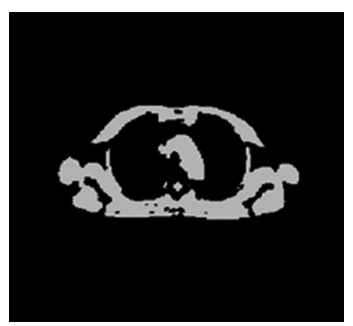

Mask_fat

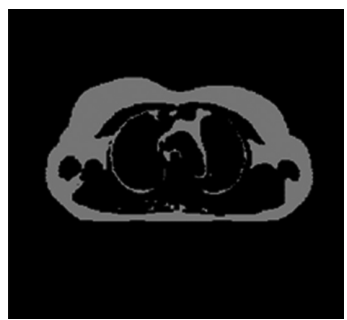

Original dCT

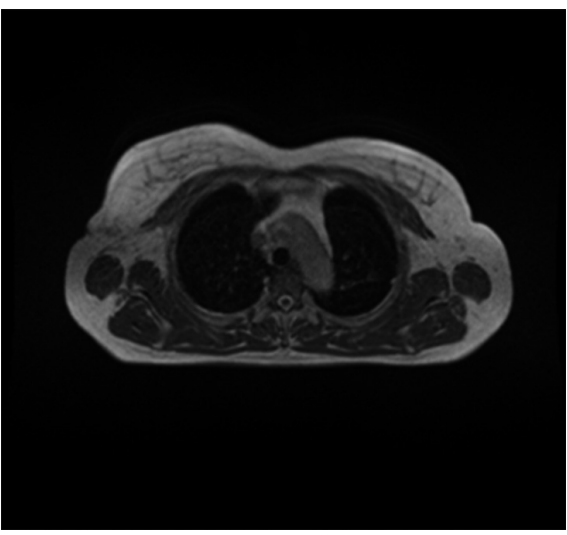

Mask_lung

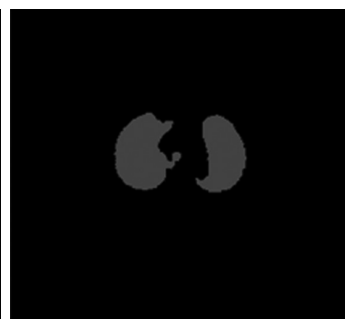

Synthesized CT

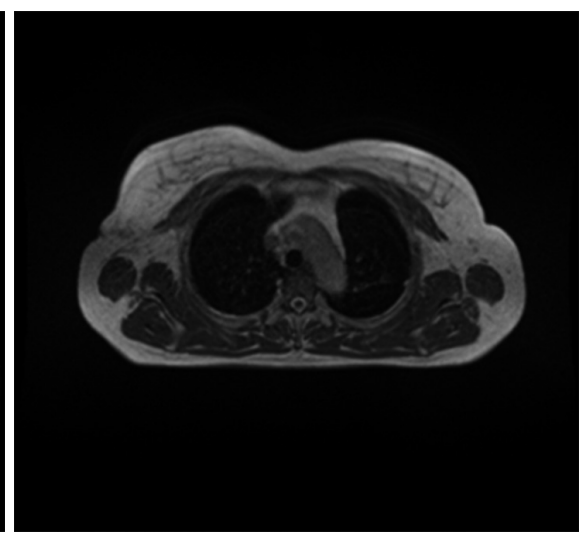

Mask_bone

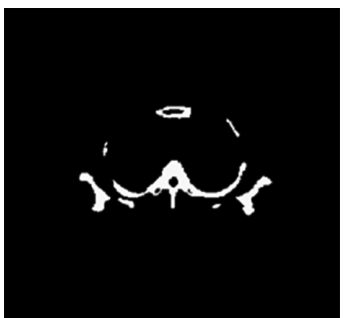

Mask_air

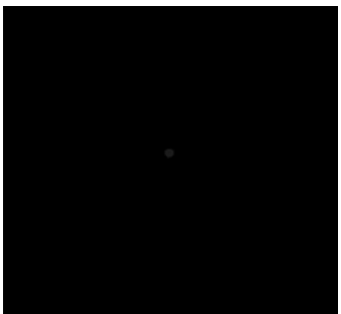

Fig. 4. Combine the mask images generated through the five deep-running models to generate the final composite CT. 
Original MR

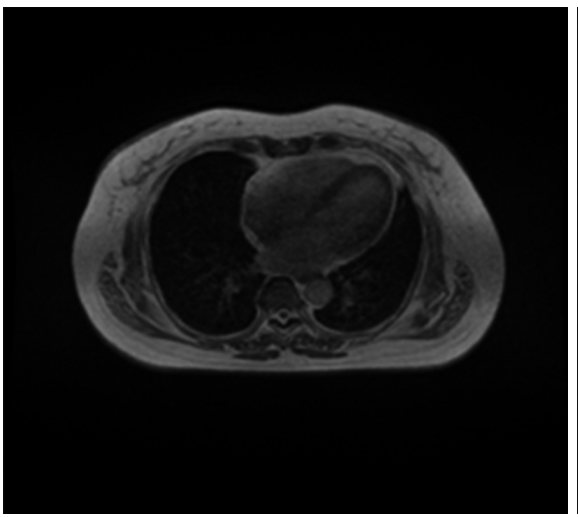

Original MR

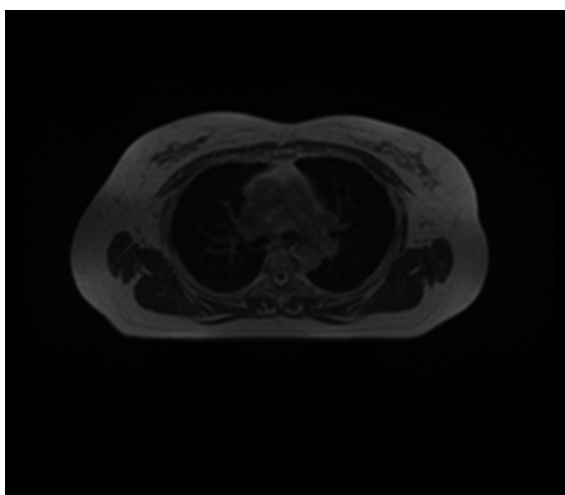

Original dCT

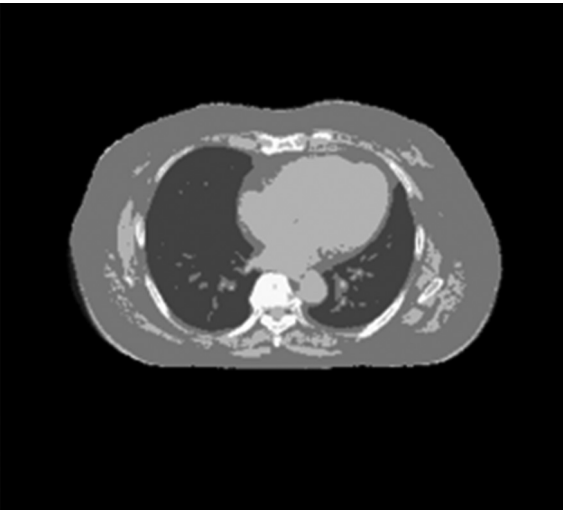

Original dCT



Synthesized CT

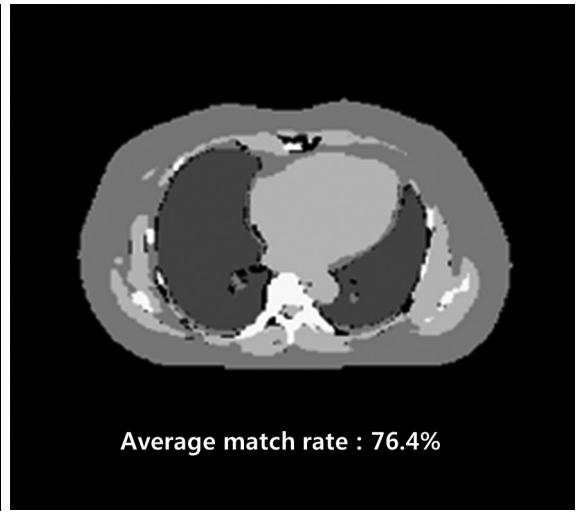

Synthesized CT

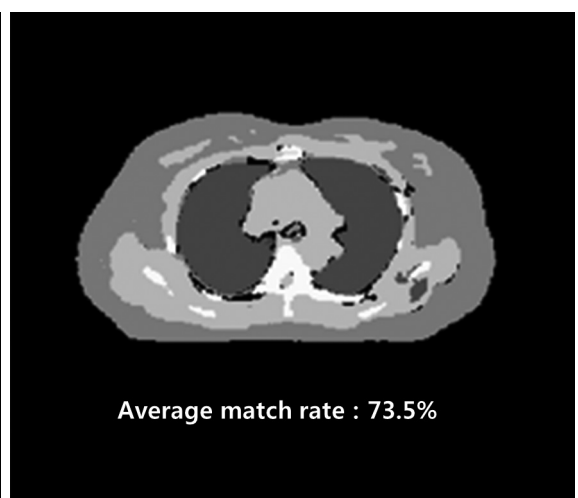

Fig. 5. Result of comparison between synthetic CT and original CT image.

Table 1. Pixel-To-Pixel Match Rate In Two Test Sets

\begin{tabular}{lccc}
\hline \multirow{2}{*}{ Patient } & \multicolumn{3}{c}{ Match rate (\%) } \\
\cline { 2 - 4 } & Maximum & Minimum & Average \\
\hline 1 & 80.3 & 67.9 & 76.4 \\
2 & 79.0 & 60.1 & 73.5 \\
\hline
\end{tabular}

dard error $0.54 \%$, median value $69.8 \%$, and the standard deviation was $4.4 \%$. The modal match rate was around $70 \%$ (range 53.7-74.9\%). The boxplot for the pixel match rate is shown in Figure 6.

The MR guided radiotherapy with daily adaptive treatment enables physicians to observe tumor reduction in realtime. This in return, leads to focusing treatment on the remaining tumors while minimizing exposure to the surrounding organs [10-13]. In the case of head and neck cancer, the tumor size dramatically changes during treatment, and real time adaptive MR guided radiotherapy can further reduce normal tissue dose, which would eventually decrease adverse events. It has been reported that the patient reported pain is reduced, while tumor control was maintained [14]. In

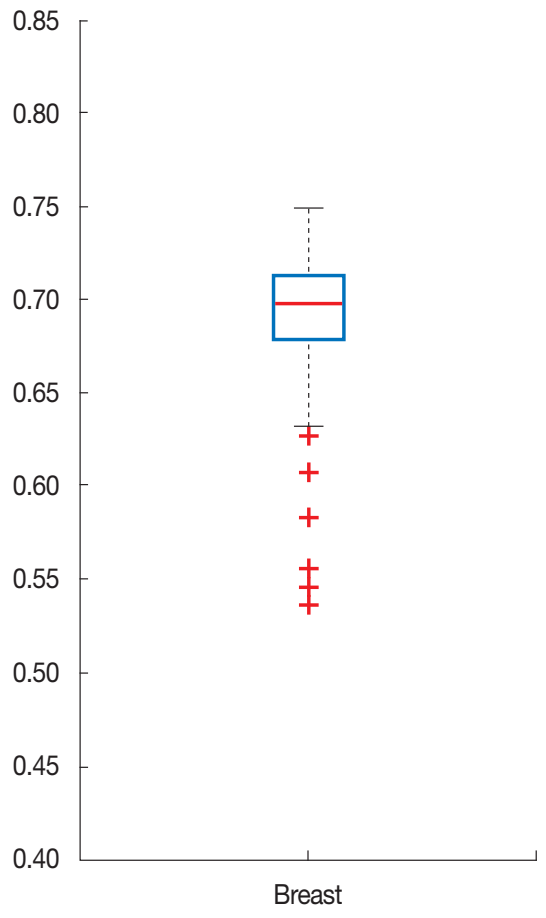

Fig. 6. Overall patient test results, pixel match rate. 
this respect, MR guided radiotherapy is the latest treatment technique capable of real-time verification of volumetric dose distribution. But there is a limit to the deformable registration algorithm due to the volume changes during image transformation process, which could then lead to inaccurate calculation of radiation dose distribution. Although quality assurance methods have been proposed to overcome these dose calculation errors, ultimate solutions require the acquisition of CT image, simultaneously. However, acquiring both CT and MR image simultaneously is currently not feasible. Furthermore, repeated CT would result in increased radiation exposure to the patient. Thus, generation of synthetic CT from real-time MR image, and not a deformed image from the baseline CT would be a superior alternative method [15]. As mentioned above, currently available real time adaptive plan could not calculate accurate radiation dose due to volume changes made during deformable registration process [4]. In this study, we constructed and tested a synthetic CT generation algorithm from MR image acquired during treatment using deep learning method. Independent training and validation with larger cohort for multiple sites is currently underway.

U-net model was chosen as a deep learning method as it is much less time-consuming compared to the atlas based manual segmentation method. Besides, the atlas based manual matching is not easily applied in the analysis after the training set. Further, U-net model would have a better potential for future development of synthetic CT generation algorithms. Recently, a deep convolutional neural network method for synthetic CT generation has been presented [5]. Notable mean absolute errors and results were presented, as well as significant results compared to deformable atlas registration and patch-based atlas fusion.

As already mentioned, it is expected that more accurate dose calculation would be possible through the development of synthetic CT generation algorithm. In this study, the overall match rate was $60-70 \%$ and it is expected that the accuracy and analytical power will improve through analysis using more input data. To increase the match rate for dCT, Five -level division by Hounsfield Unit was used instead of dCT, itself in the current study. But it is requisite to review the correct classification manually as boundaries may be quite ambiguous. Precise boundary segmentation between organs and tissues is an important part to increase the match rate. Image review for the appropriateness of applied segmentation in the training set by physician or trained researcher would further improve accuracy. However, it is difficult to set exact segmentation points, and classification by physician or trained researcher is cumbersome and requires a great deal of research resources. Based on the result of this preliminary study, the match rate of transformation between bone and air was low in deep learning based synthetic CT generation. When using synthetic CT in radiotherapy, we need a model that can best classify the bone and air from other tissues, which are more or less water equivalent in terms of electron density. If the synthetic CT generation algorithm model can accurately distinguish bone and air, we could further reduce the error in the radiation treatment planning. With these results, we will be able to predict delivered radiation doses more accurately.

Three areas requiring further exploitation was revealed through this study. First, we should evaluate the feasibility of MR image integration and fit of required MR image modification for real-time MR image guided radiotherapy. Secondly, further refinement and tuning is required for automated segmentation procedure of dCT using Hounsfield unit, especially for bone and air. Lastly, the reliability of generated dCT to be used should be given second thought as dCT cannot be free from all the aforementioned pitfalls. Thus using $\mathrm{dCT}$ as gold standard could set bars on the ability of algorithm in training.

Significance of this study is that though preliminary in design, this work ha s shown the possibility of deep learning based synthetic CT generation by evaluation of image characteristics of MR and CT. Capturing the difference of image characteristics between bone, air and other tissues is an important part of synthetic CT generation.

\section{Conclusion}

From the acquired MR and CT images, we developed and evaluated the deep learning model for efficient synthetic CT generation. Algorithm optimization will be done through the process of synthetic CT generation and evaluation of different body parts using deep learning model with additional data from more patients. In the future, it is expected that the MR image database will be built for various parts of the body, such as abdomen, thorax, and pelvis. Next step of the research would focus on demonstrating the feasibility of adapting the deep learning model to the rest of the body area beyond thorax. 


\section{Acknowledgements}

This work was supported by the National Research Foundation of Korea (NRF) grant funded by the Korean government (MSIP) (No. 2017M2A2A7A02020640).

\section{References}

1. Park JM, Park SY, Kim HJ, Wu HG, Carlson J, Kim JI. A comparative planning study for lung SABR between tri-Co-60 magnetic resonance image guided radiation therapy system and volumetric modulated arc therapy. Radiother. Oncol. 2016;120(2):279285.

2. Choi CH, Park SY, Kim JI, Kim JH, Kim K, Carlson J, Park JM. Quality of tri-Co-60 MR-IGRT treatment plans in comparison with VMAT treatment plans for spine SABR. Br. J. Radiol. 2017;90(1070): 20160652.

3. Khoo VS, Joon DL. New developments in MRI for target volume delineation in radiotherapy. Br. J. Radiol. 2006;79(1):S2-S15.

4. Schultheiss TE, Tome WA, Orton CG. Point/counterpoint: it is not appropriate to "deform" dose along with deformable image registration in adaptive radiotherapy. Med. Phys. 2012;39(11): 6531-6533.

5. Han X. MR-based synthetic CT generation using a deep convolutional neural network method. Med. Phys. 2017;44(4):14081419.

6. Dreiseitl S, Ohno-Machado L, Kittler H, Vinterbo S, Billhardt H, Binder M. A comparison of machine learning methods for the diagnosis of pigmented skin lesions. J. Biomed. Inform. 2001;34(1): 28-36.

7. Wei L, Yang Y, Nishikawa R, Jiang Y. A Study on Several Machine-
Learning Methods for Classification of Malignant and Benign Clustered Microcalcifications. IEEE Trans. Med. Imaging. 2005; 24(3):371-380.

8. Jones N. Computer science: The learning machines. Nature. 2014; 505(7482):146-148.

9. Ronneberger O, Fischer P, Brox T. U-net: Convolutional networks for biomedical image segmentation. International Conference on Medical image computing and computer-assisted intervention. Springer, Cham. 2015:234-241.

10. Seregni M, Paganelli C, Summers P, Bellomi M, Baroni G, Riboldi M. A Hybrid Image Registration and Matching Framework for Real-Time Motion Tracking in MRI-Guided Radiotherapy. IEEE Trans. Biomed. Eng. 2018;65(1):131-139.

11. McPartlin AJ, et al. MRI-guided prostate adaptive radiotherapy A systematic review. Radiother. Oncol. 2016;119(3):371-380.

12. Vestergaard A, et al. The potential of MRI-guided online adaptive re-optimisation in radiotherapy of urinary bladder cancer. Radiother. Oncol. 2016;118(1):154-159.

13. Abedi I, Tavakkoli MB, Jabbari K, Amouheidari A, Yadegarfard G. Dosimetric and Radiobiological Evaluation of Multiparametric MRI-Guided Dose Painting in Radiotherapy of Prostate Cancer. J. Med. Signals Sens. 2017;7(2):114-121.

14. Jeon SH, Shin KH, Park SY, Kim JI, Park JM, Kim JH, Chie EK, Wu $H$. Seroma changes during magnetic resonance imaging-guided partial breast irradiation and its clinical implications. Radiat. Oncol. 2017;12(1):103.

15. Chen AM, Hsu S, Lamb J, Yang Y, Agazaryan N, Steinberg ML, Low DA, Cao M. MRI-guided radiotherapy for head and neck cancer: initial clinical experience. Clin. Transl. Oncol. 2018;20(2):160-168.

16. Lee HJ, Kadbi M, Bosco G, Ibbott GS. Real-time volumetric relative dosimetry for magnetic resonance-image-guided radiation therapy (MR-IGRT). Phys. Med. Biol. 2018;63(4):045021. 